NUC-MINN-00/14-T

\title{
Relativistic Viscous Fluid Description of Microscopic Black Hole Wind
}

\author{
J. I. Kapusta* \\ School of Physics and Astronomy \\ University of Minnesota \\ Minneapolis, MN 55455
}

(October 30, 2018)

\begin{abstract}
Microscopic black holes explode with their temperature varying inversely as their mass. Such explosions would lead to the highest temperatures in the present universe, all the way to the Planck energy. Whether or not a quasi-stationary shell of interacting matter undergoing radial hydrodynamic expansion surrounds such black holes is controversial. In this paper relativistic viscous fluid equations are applied to the problem assuming sufficient particle interaction. It is shown that a self-consistent picture emerges of a fluid just marginally kept in local thermal equilibrium; viscosity is a crucial element of the dynamics.
\end{abstract}

PACS numbers: 04.70.Dy, 11.10.Wx, 26.50.+x

*kapusta@physics.spa.umn.edu 
Hawking radiation from black holes [1] is of fundamental interest because it relies on the application of relativistic quantum field theory in the presence of the strong field limit of gravity, a so far unique situation. It is also of great interest because of the temperatures involved. A black hole with mass $M$ radiates thermally with a temperature

$$
T_{h}=\frac{m_{\mathrm{P}}^{2}}{8 \pi M}
$$

where $m_{\mathrm{P}}=G^{-1 / 2}=1.22 \times 10^{19} \mathrm{GeV}$ is the Planck mass. (Units are $\hbar=c=k_{\mathrm{B}}=1$.) In order for the black hole to evaporate it must have a temperature greater than that of the present-day black-body radiation of the universe of $2.7 \mathrm{~K}=2.3 \times 10^{-4} \mathrm{eV}$. This implies that $M$ must be less than $1 \%$ of the mass of the Earth, hence the black hole most likely would have been formed primordially and not from stellar collapse. The black hole temperature eventually goes to infinity as its mass goes to zero, although once $T_{h}$ becomes comparable to the Planck mass the semi-classical calculation breaks down and the regime of full quantum gravity is entered. Only in two other situations are such enormous temperatures achievable: in the early universe ( $T$ similarly asymptotically high) and in central collisions of heavy nuclei like gold or lead ( $T=500 \mathrm{MeV}$ is expected at the RHIC (Relativistic Heavy Ion Collider) just completed at Brookhaven National Laboratory and $T=1 \mathrm{GeV}$ is expected at the LHC (Large Hadron Collider) at CERN to be completed in 2005). The spontaneously broken chiral symmetry of QCD gets restored in a phase transition/rapid crossover at a temperature around $160 \mathrm{MeV}$, while the spontaneously broken gauge symmetry in the electroweak sector of the standard model gets restored in a phase transition/rapid crossover at a temperature around $100 \mathrm{GeV}$. The fact that temperatures of the latter order of magnitude will never be achieved in a terrestrial experiment motivates me here to study the fate of primordial black holes during the final minutes of their lives when their temperatures have risen to $100 \mathrm{GeV}$ and above. The fact that primordial black holes have not yet been observed [2] does not deter me in the least.

There has been some controversy over whether the particles scatter from each other after being emitted, perhaps even enough to allow a fluid description of the wind coming from 
the black hole. First the situation will be reviewed, and then it will be shown that a selfconsistent description is afforded by the application of relativistic viscous fluid equations, at least towards the end of the black hole's existence and under certain assumptions.

When $T_{h} \ll m_{\mathrm{e}}$ (electron mass) only photons, gravitons, and neutrinos will be created with any significant probability. These particles will not interact with each other but will be emitted into the surrounding space with the speed of light. Even when $T_{h} \approx m_{\mathrm{e}}$ the Thomson cross section is too small to allow the photons to scatter very frequently in the rarified electron-positron plasma around the black hole. This may change when $T_{h} \approx 100 \mathrm{MeV}$ when muons and charged pions are created in abundance. At somewhat higher temperatures hadrons are copiously produced and local thermal equilibrium may be achieved, although exactly how is an unsettled issue. Are hadrons emitted directly by the black hole? If so, they will be quite abundant at temperatures of order $150 \mathrm{MeV}$ because their mass spectrum rises exponentially (Hagedorn growth as seen in the Particle Data Tables [3]). Because they are so massive they move nonrelativistically and may form a very dense equilibrated gas around the black hole. But hadrons are composites of quarks and gluons, so perhaps quarks and gluon jets are emitted instead? These jets must decay into the observable hadrons on a typical length scale of $1 \mathrm{fm}$ and a typical time scale of $1 \mathrm{fm} / \mathrm{c}$. Once the hadrons appear they may form an equilibrated gas around the black hole just as if they had been produced directly albeit with some time delay. One can find arguments both for [4] and against [5] thermal equilibrium among the strongly interacting hadrons outside the Schwarzschild radius. Recently a numerical study of photosphere formation around a primordial black based on a relaxation time approximation to the Boltzmann equation has been performed for QED and QCD interactions [6]. It was found that significant particle scattering would lead to a photosphere though not perfect fluid flow. Certainly this is a very difficult and open problem in quantum statistical mechanics, just as it is in high energy heavy ion collisions |7.

Let us assume that a primordial black hole is surrounded by a shell of expanding interacting matter in approximately local thermal equilibrium when $T_{h}$ is large enough. A 
detailed description of how this situation comes to be is a difficult problem as discussed above and is not addressed in this paper. The relativistic imperfect fluid equations describing a steady-state, spherically symmetric flow with no net baryon number or electric charge and neglecting gravity (see below) are $T_{; \nu}^{\mu \nu}=$ black hole source. The nonvanishing components of the energy-momentum tensor in radial coordinates are [8]

$$
\begin{aligned}
& T^{00}=\gamma^{2}(P+\epsilon)-P+v^{2} \Delta T_{\text {diss }} \\
& T^{0 r}=v \gamma^{2}(P+\epsilon)+v \Delta T_{\text {diss }} \\
& T^{r r}=v^{2} \gamma^{2}(P+\epsilon)+P+\Delta T_{\text {diss }}
\end{aligned}
$$

representing energy density, radial energy flux, and radial momentum flux, respectively, in the rest frame of the black hole. Here $v$ is the radial velocity with $\gamma$ the corresponding Lorentz factor, $u=v \gamma, \epsilon$ and $P$ are the local energy density and pressure, and

$$
\Delta T_{\mathrm{diss}}=-\frac{4}{3} \eta \gamma^{2}\left(\frac{d u}{d r}-\frac{u}{r}\right)-\zeta \gamma^{2}\left(\frac{d u}{d r}+\frac{2 u}{r}\right)
$$

where $\eta$ is the shear viscosity and $\zeta$ is the bulk viscosity. A thermodynamic identity gives $T s=P+\epsilon$ for zero chemical potentials, where $T$ is temperature and $s$ is entropy density. There are two independent differential equations of motion to solve for the functions $T(r)$ and $v(r)$.

An integral form of these equations is probably more useful since it can readily incorporate the input luminosity $L_{i}$ from the black hole. The first represents the equality of the energy flux passing through a sphere of radius $\mathrm{r}$ with the luminosity of the black hole.

$$
4 \pi r^{2} T^{0 r}=L_{i}
$$

The second follows from integrating a linear combination of the differential equations. It represents the combined effects of the entropy from the black hole together with the increase of entropy due to viscosity.

$$
4 \pi r^{2} u s=4 \pi \int_{r_{i}}^{r} d r^{\prime} r^{\prime 2} \frac{1}{T}\left[\frac{8}{9} \eta\left(\frac{d u}{d r^{\prime}}-\frac{u}{r^{\prime}}\right)^{2}+\zeta\left(\frac{d u}{d r^{\prime}}+\frac{2 u}{r^{\prime}}\right)^{2}\right]+\frac{L_{i}}{T_{h}}
$$


The term $L_{i} / T_{h}$ arises from equating the entropy per unit time lost by the black hole $-d S_{\mathrm{h}} / d t$ with that flowing into the matter. Using the area formula for the entropy of a black hole, $S_{\mathrm{h}}=m_{\mathrm{P}}^{2} \pi r_{S}^{2}=4 \pi M^{2} / m_{\mathrm{P}}^{2}$, and identifying $-d M / d t$ with the luminosity, the entropy input from the black hole is obtained.

The above pair of equations are to be applied beginning at some radius $r_{i}$ greater than the Schwarzschild radius $r_{S}$, that is, outside the quantum particle production region of the black hole. The radius $r_{i}$ at which the imperfect fluid equations are first applied should be chosen to be greater than the Schwarzschild radius, otherwise the computation of particle creation by the black hole would be invalid. It should not be too much greater, otherwise particle collisions would create more entropy than is accounted for by eq. (5). The energy and entropy flux into the fluid come from quantum particle creation by the black hole at temperature $T_{h}$, and particle production is dominated by particles with mass less than the temperature. Massless particles emitted from a surface at rest have an average outward velocity of $1 / \sqrt{3}$. Therefore it is not unreasonable to assume the initial flow velocity is $v_{i}=v\left(r_{i}\right)=1 / \sqrt{3}$. Furthermore, considerations of smoothness and continuity suggest that $d v / d r=0$ at $r_{i}$. In fact one should not expect the precise choice of initial conditions to matter at large radii; see the discussion of the scaling solutions later in this paper. Once the functions $s(T), \eta(T)$, and $\zeta(T)$ are specified, $r_{i}$ and $T_{i}=T\left(r_{i}\right)$ can be determined by the integral form of the equations of motion, eqs. (4) and (5). Gravitational effects are of order $r_{S} / r$, hence negligible for $r>(5-10) r_{S}$.

A black hole has a Schwarzschild radius $r_{S}=2 M / m_{\mathrm{P}}^{2}=1 / 4 \pi T_{h}$. Note that $\pi T_{h} \cdot 2 r_{S}=$ $1 / 2$. Roughly, the average thermal momentum of a massless particle times the diameter of the black hole is $1 / 2$. This is just a manifestation of the uncertainty principle applied to the creation of an excitation in a confined region of space. The luminosity is

$$
L=-\frac{d M}{d t}=\alpha(M) \frac{m_{\mathrm{P}}^{4}}{M^{2}}=64 \pi^{2} \alpha\left(T_{h}\right) T_{h}^{2}
$$

where $\alpha(M)$ is a function reflecting the species of particles available for creation in the gravitational field of the black hole. It is generally sufficient to consider only those particles with 
mass less than $T_{h}$; more massive particles are exponentially suppressed by the Boltzmann factor. Then

$$
\alpha=2.011 \times 10^{-8}\left[4200 N_{0}+2035 N_{1 / 2}+835 N_{1}+95 N_{2}\right] .
$$

Here $N_{s}$ is the net number of polarization degrees of freedom for all particles with spin $s$ and with mass less than $T_{h}$. The coefficients for spin 1/2, 1 and 2 were computed in ref. [9] and for spin 0 in ref. [10]. In the standard model $N_{0}=4$ (Higgs), $N_{1 / 2}=90$ (three generations of quarks and leptons), $N_{1}=24(\mathrm{SU}(3) \times \mathrm{SU}(2) \times \mathrm{U}(1)$ gauge theory), and $N_{2}=2$ (gravitons). This assumes $T_{h}$ is greater than the temperature for the electroweak gauge symmetry restoration [11]. Numerically $\alpha\left(T_{h}>100 \mathrm{GeV}\right)=4.43 \times 10^{-3}$. Starting with a black hole of temperature $T_{h}$, the time it takes to evaporate/explode is

$$
\Delta t=\frac{m_{\mathrm{P}}^{2}}{3 \alpha\left(T_{h}\right)\left(8 \pi T_{h}\right)^{3}} .
$$

This is also the characteristic time scale for the rate of change of the luminosity of a black hole with temperature $T_{h}$. Generally $\Delta t \gg 1 / T_{h}$, thus justifying the quasi-stationary assumption [12]. For example, a black hole with temperature $1 \mathrm{TeV}$ has a Schwarzschild radius of $1.57 \times 10^{-5} \mathrm{fm}$, a mass of $10^{10} \mathrm{~g}$, a luminosity of $7 \times 10^{27} \mathrm{erg} / \mathrm{s}$, and has 464 seconds to live. The input luminosity to the expanding fluid $L_{i}$ will be less than the total luminosity $L$, because gravitons will escape without scattering, and neutrinos could scatter only in those regions where the temperature is greater than about $100 \mathrm{GeV}$. The reason is neutrino cross sections above $100 \mathrm{GeV}$ will be similar to the cross sections of other fermions because of the restoration of the spontaneously broken electroweak gauge symmetry.

Determination of the equation of state as well as the two viscosities for temperatures ranging from $\mathrm{MeV}$ to $\mathrm{TeV}$ and more is a formidable task. Here we shall consider two interesting limits and then a semi-realistic situation. A realistic, quantitative description of the relativistic black hole wind, including the asymptotic observed particle spectra, is left for a future publication.

First, consider the adiabatic limit (like milk) with an equation of state $\epsilon=a T^{4}, s=$ $(4 / 3) a T^{3}$, and $\eta=\zeta=0$. This is equivalent to assuming that the mean free paths of the 
particles are all small compared to the length scale over which the temperature and other thermodynamic quantities change significantly. A scaling solution, valid when $\gamma \gg 1$, is $T(r)=T_{0}\left(r_{0} / r\right)$ and $\gamma(r)=\gamma_{0}\left(r / r_{0}\right)$, where $\gamma_{0} T_{0}=T_{h}$. The $r_{0}$ is any reference radius satisfying the stated criterion.

Second, consider the highly viscous isoergic limit (like honey) in the sense that the flow velocity approaches a limiting value $v_{0}$ at large $r$. This requires a power-like equation of state $\epsilon \propto T^{\delta}$ and viscosities $\eta \propto \zeta \propto T^{\delta / 2}$. It results in the scaling solution $T(r)=T_{0}\left(r_{0} / r\right)^{2 / \delta}$. This is not very realistic: a massless gas with dimensionless coupling constants and $\delta=4$ would require viscosities of order $T^{2}$ whereas one would expect $T^{3}$ on dimensional grounds.

Now consider a semi-realistic situation with $\epsilon=a T^{4}, s=(4 / 3) a T^{3}, \eta=b_{S} T^{3}$, and $\zeta=$ $b_{B} T^{3}$. This is typical of relativistic gases with dimensionless coupling constants, although quantum effects will give logarithmic corrections [14,13]. A scaling solution, valid at large radii when $\gamma \gg 1$, is $T(r)=T_{0}\left(r_{0} / r\right)^{2 / 3}$ and $\gamma(r)=\gamma_{0}\left(r / r_{0}\right)^{1 / 3}$. The constants are related by $36 a T_{0} r_{0}=\left(32 b_{S}+441 b_{B}\right) \gamma_{0}$. This $r$-dependence of $T$ and $\gamma$ is exactly what was conjectured in ref. [4].

Is the semi-realistic situation described above really possible? Can approximate local thermal equilibrium, if achieved, be maintained? The requirement is that the inverse of the local volume expansion rate $\theta=u_{; \mu}^{\mu}$ be comparable to or greater than the relaxation time for thermal equilibrium [8]. Expressed in terms of a local volume element $V$ and proper time $\tau$ it is $\theta=(1 / V) d V / d \tau$, whereas in the rest frame of the black hole the same quantity can be expressed as $\left(1 / r^{2}\right) d\left(r^{2} u\right) / d r$. Explicitly

$$
\theta=\frac{7 \gamma_{0}}{3 r_{0}}\left(\frac{r_{0}}{r}\right)^{2 / 3}=\frac{7 \gamma_{0}}{3 r_{0} T_{0}} T .
$$

Of prime importance in achieving and maintaining local thermal equilibrium in a relativistic plasma are multi-body processes such as $2 \rightarrow 3$ and $3 \rightarrow 2$, etc. This has been well-known when calculating quark-gluon plasma formation and evolution in high energy heavy ion collisions [15] and has been emphasized in ref. [4] in the context of black hole evaporation. This is a formidable task in the standard model with its 16 species of particles. Instead 
we make three estimates for the requirement that local thermal equilibrium be maintained. The first and simplest estimate is to require that the thermal DeBroglie wavelength of a massless particle, $1 / 3 T$, be less than $1 / \theta$. The second estimate is to require that the Debye screening length for each of the gauge groups in the standard model be less than $1 / \theta$. The Debye screening length is the inverse of the Debye screening mass $m_{n}^{\mathrm{D}}$ where $n=1,2,3$ for the gauge groups $\mathrm{U}(1), \mathrm{SU}(2), \mathrm{SU}(3)$. Generically $m_{n}^{\mathrm{D}} \propto g_{n} T$ where $g_{n}$ is the gauge coupling constant and the coefficient of proportionality is essentially the square root of the number of charge carriers [16]. For example, for color $\mathrm{SU}(3) m_{3}^{\mathrm{D}}=g_{3} \sqrt{1+N_{\mathrm{f}} / 6} T$ where $N_{\mathrm{f}}$ is the number of light quark flavors at the temperature $T$. The numerical values of the gauge couplings are: $g_{1}=0.344, g_{2}=0.637$, and $g_{3}=1.18$ (evaluated at the scale $m_{Z}$ ) [3]. So within a factor of about 2 we have $m^{\mathrm{D}} \approx T$. The third and most relevant estimate is the mean time between two-body collisions in the standard model for temperatures greater than the electroweak symmetry restoration temperature. This mean time was calculated in ref. [14] in the process of calculating the viscosity in the relaxation time approximation. Averaged over all particle species in the standard model one may infer from that paper an average time of $3.7 / T$. Taking into account multi-body reactions would decrease that by about a factor of two to four. All three of these estimates are consistent within a factor of 2 or 3 . The conclusion to be drawn is that local thermal equilibrium should be achieved when $\theta \lesssim T$, or $84 a /\left(32 b_{S}+441 b_{B}\right) \lesssim 1$. This criterion places a minimum value on the strength of the viscosities. Once thermal equilibrium is achieved it is not lost because $\theta / T$ is independent of $r$. The picture that emerges is that of an imperfect fluid just marginally kept in local equilibrium by viscous forces.

The hot shell of matter surrounding a primordial black hole provides a theoretical testing ground rivaled only by the big bang itself. In addition to the questions already raised, one may contemplate baryon number violation at high temperature and how physics beyond the standard model might be important in the last few minutes in the life of a primordial black hole. Finally, such black holes may contribute to the highest energy cosmic rays whose origin is a long-standing puzzle. Experimental discovery of exploding black holes will be one 
of the great challenges in the new millennium.

\section{ACKNOWLEDGEMENTS}

I am grateful to G. Amelino-Camelia and A. Heckler for many illuminating discussions and to P. Ellis for comments on the manuscript. This work was supported by the US Department of Energy under grant DE-FG02-87ER40328. 


\section{REFERENCES}

[1] S. W. Hawking, Nature (London) 248, 30 (1974); Commun. Math. Phys. 43, 199 (1975).

[2] B. J. Carr and J. H. MacGibbon, Phys. Rep. 307, 141 (1998).

[3] Particle Data Group: Review of Particle Physics, Eur. Phys. J. C3, 1 (1998).

[4] A. F. Heckler, Phys. Rev. D 55, 480 (1997); Phys. Rev. Lett. 78, 3430 (1997).

[5] J. Oliensis and C. T. Hill, Phys. Lett. B143, 92 (1984); J. H. MacGibbon and B. R. Webber, Phys. Rev. D 41, 3052 (1990); J. H. MacGibbon and B. J. Carr, Astrophys. J. 371, 447 (1991); F. Halzen, E. Zas, J. H. MacGibbon and T. C. Weekes, Nature (London) 353, 807 (1991).

[6] J. Cline, M. Mostoslavsky, and G. Servant, Phys. Rev. D 59, 063009 (1999).

[7] See, for example, the proceedings of the Quark Matter series of international conferences, the most recent in print being: Proceedings of Quark Matter '99, Nucl. Phys. A661, (1999).

[8] C. W. Misner, K. S. Thorne, and J. A. Wheeler, Gravitation (W. H. Freeman and Company, San Francisco, 1973).

[9] D. N. Page, Phys. Rev. D 13, 198 (1976).

[10] N. Sanchez, Phys. Rev. D 18, 1030 (1978); the coefficient was extracted from figure 5 of this paper to within about $5 \%$ accuracy.

[11] One may usefully contemplate whether the helicity-0 component of the $\mathrm{W}$ and $\mathrm{Z}$ bosons below the symmetry restoring temperature is to be treated as the third component of a free massive vector boson or as a free massive scalar particle on account of the Higgs mechanism. If the vexing problem of quark/gluon versus hadron production has not attracted the curiosity of the reader this ought to.

[12] J. H. MacGibbon, Phys. Rev. D 44, 376 (1991). 
[13] G. Baym, H. Monien, C. J. Pethick, and D. G. Ravenhall, Phys. Rev. Lett. 64, 1867 (1990).

[14] M. E. Carrington and J. I. Kapusta, Phys. Rev. D 47, 5304 (1993).

[15] K. Kinder-Geiger, Phys. Rep. 258, 237 (1995); S.M.H. Wong, Phys. Rev. D 54, 2588 (1996).

[16] J. I. Kapusta, Finite Temperature Field Theory (Cambridge University Press, Cambridge, England, 1989). 